\title{
A Cognitive System Design for Mobile Robot Based on an Intelligent Algorithm
}

\author{
Ahmed S. Al-Araji ${ }^{1}$, Attarid K. Ahmed ${ }^{2}$ \\ ${ }^{1,2}$ Control and Systems Eng. Dept. at University of Technology \\ 60166@uotechnology.edu.iq
}

\begin{abstract}
This paper presents a cognitive system based on a nonlinear Multi-Input MultiOutput (MIMO) Proportion Integral Derivative (PID) Modified Elman Neural Network (MENN) controller and the Square Road Map (SRM) method to guide the mobile robot during the continuous path-tracking with collision-free navigation through static obstacles. The proposed cognitive system consists of two parts: the first part is to plan the desired path for the mobile robot with the static obstacle environment in order to determine the target point and to avoid the obstacles based on the proposed square road map algorithm. The second part is to guide and track the wheeled mobile robot on the desired path equation based on the proposed nonlinear MIMO-PID-MENN controller with the intelligent algorithm. The Particle Swarm Optimization (PSO) is used to on-line tune the variable control parameters of the proposed controller to get the optimal torques actions for the mobile robot platform. Based on using the MATLAB package (2017), the numerical simulation results show that the proposed cognitive system has high accuracy for planning the desired path equation in terms of avoiding the static obstacles with smooth and short distance and generating a perfect torque action of $(0.7 \mathrm{~N} . \mathrm{m})$ without a saturation state of (3.07 N.m), which leads to minimize the tracking pose error for the mobile robot to the zero value approximation. These results were confirmed by a comparative study with different nonlinear PID controller types in terms of number of iterations and the performance index.
\end{abstract}

Index Terms - Mobile Robot, Modified Elman Neural Network, Path Planning, PID controller, PSO Algorithm.

\section{INTRODUCTION}

In general, the term of path-planning is a mission of a mobile robot navigation in a work space with obstacles avoidance. The specifications of the optimal paths should be made such that they minimize the number of turnings and avoid small rotation radii, which in turn reduce the amount of braking and lead to obtain the shortest distance between the start and the target points [1]. Therefore, the motion control for the mobile robot should track and execute the path planning because there are many applications in various life fields such as science, education, industry, mining, entertainment, security and military which need the wheeled mobile robot system. Based on this importance, the mobile robot is still an active research area [2]. In recent years, different types of evolutionary techniques such as Genetic Algorithm [3], Ant Colony Optimization [4] and Particle Swarm Optimization (PSO) [5] have been widely used for the path planning and for solving the problems of static and dynamic obstacles in the environment for various tasks. In addition to that, many types of control algorithms have been designed based on the mobile robot's mathematical model. These control algorithms have been proposed to solve the mobile robot motion control to follow the desired path with high performance of the controller in terms of generating an optimal control 
action that leads to minimizing the tracking pose error during tracking the desired path. These algorithms include the nonlinear neural PID controller [6], fuzzy logic and PID controllers [7], neural networks controllers [8 and 9], adaptive fuzzy with back-stepping controllers [10], adaptive sliding mode controllers [11], neural predictive controllers [12], [13], and nonlinear fractional order PID neural controllers [14], [15]. The motivation for this research is to find the best path with obstacles avoidance and to track and stabilize the mobile robot on the desired path by generating the best action without undesirable states such as a saturation state and a spike action state. After a comparative study with different nonlinear PID controllers, the contributions of this paper are illustrated as follows: the cognition desired path equation can generate an optimal path with high computational accuracy based on the square road map algorithm in order to achieve the minimum distance with obstacles avoidance; the modified Elman neural network based PID control equation with the PSO algorithm has derived the control law in order to generate the best torque action and to follow the desired path equation; tracking different desired path equations to confirm the capability of the proposed cognitive system through reducing the pose error of the mobile robot platform; investigating the robustness performance by adding bounded disturbances to the output of the proposed controller; and verifying the adaptation performance when changing the initial pose state of the mobile robot platform.

The organization of this paper can be described as follows: Section two describes the dynamic wheeled mobile robot model. Section three derives the proposed cognitive system. Section four presents the numerical simulation results of the cognitive system in an environment. In section five, the conclusions are drawn.

\section{NON-HOLONOMIC WHEELED MOBILE ROBOT MODEL}

In general, the platform of the wheeled mobile robot consists of two DC motors which drive the left and right wheels of the mobile robot and stabilize the platform. One castor wheel is used in the front of the cart [1] as shown in Fig. 1.

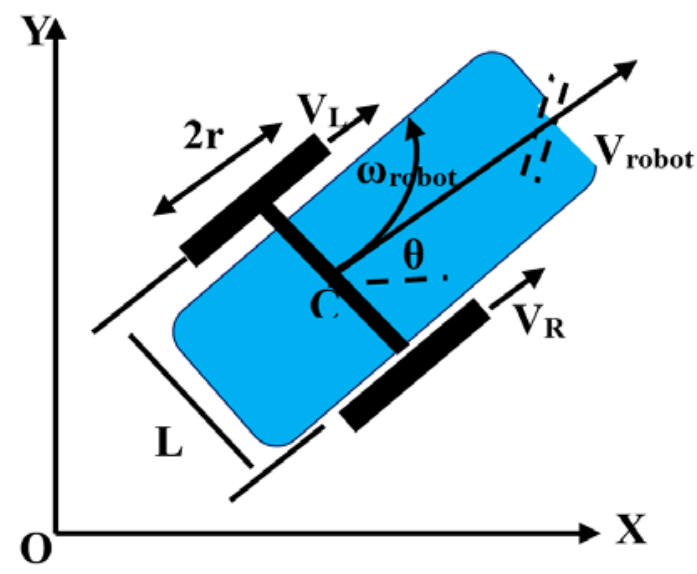

FIG. 1. THE MOBILE ROBOT PLATFORM MODEL.

The aim of these two DC motor actuators for left and right wheels is to move and orient the robot platform. The two wheels have the same radius $r, L$ is the distance between the left and right wheels, and the centre of the gravity is denoted as $c$ for the mobile robot.

The $[\mathrm{O}, \mathrm{X}, \mathrm{Y}]$ is defined as the global coordinate frame, while $\mathrm{q}$ is defined as the pose vector of the local coordinate of the mobile robot as in equation (1): 


$$
q=(x, y, \theta)^{T}
$$

Where, the $(\mathrm{x}, \mathrm{y})$ is denoted as the position coordinates at point $c ; \theta$ is denoted as the orientation angle.

To confirm that the wheeled mobile robot has the ability for motion and orientation, two main conditions should be achieved: pure-rolling for each wheel; and non-slipping for each wheel to put the lateral velocity of the mobile robot equal to zero as equation (2) [6].

$$
\dot{x}(t) \sin \theta(t)=y(t) \cos \theta(t)
$$

The kinematics model of the wheeled mobile robot is represented as follows in the global frame [8]:

$$
\begin{aligned}
& \dot{\theta}(t)=\frac{r(\omega r(t)-\omega l(t))}{L} \\
& \dot{y}(t)=\frac{r(\omega r(t)-\omega l(t))}{2} \sin \theta(t) \\
& \dot{x}(t)=\frac{r(\omega r(t)-\omega l(t))}{2} \cos \theta(t)
\end{aligned}
$$

Where, $w r(t)$ and $w l(t)$ are the right and the left angular velocities, respectively.

The dynamics model of the mobile robot is represented based on the Euler Lagrange formulation, as follows [10]:

$$
\left[\begin{array}{ccc}
M & 0 & 0 \\
0 & M & 0 \\
0 & 0 & I
\end{array}\right]\left[\begin{array}{l}
\ddot{x} \\
\ddot{y} \\
\ddot{\theta}
\end{array}\right]=\frac{1}{r}\left[\begin{array}{cc}
\cos \theta & \cos \theta \\
\sin \theta & \sin \theta \\
\frac{L}{2} & \frac{-L}{2}
\end{array}\right]\left[\begin{array}{l}
\tau_{L} \\
\tau_{R}
\end{array}\right]-\left[\begin{array}{l}
\tau_{d 1} \\
\tau_{d 2}
\end{array}\right]+\left[\begin{array}{c}
-\sin \theta \\
\cos \theta \\
0
\end{array}\right] \lambda
$$

Where $\tau_{L}:$ is the torque of the left wheel; $\tau_{R}:$ is the torque of the right wheel; $M$ : is the mass of the mobile robot; $I$ : is the inertia of the mobile robot; $\lambda:$ is the constraint forces; $\tau d_{1,2}:$ are the bounded dynamic disturbances.

\section{COGNITIVE SYSTEM DESIGN}

In this work, the cognitive system approach is designed based on an intelligent optimization algorithm and the square road map method. This system is used to plan a smooth desired path with a short distance to the target of the mobile robot as well as to avoid the static obstacle in the environment then to track the desired path equation by using the nonlinear MIMO-PID-MENN controller. The proposed cognitive system can be described as shown in Fig. 2. The proposed cognitive system structure consists of two layers: a) The Cognition Path Planning Layer based on the square road map algorithm; b) The Nonlinear MIMI-PIDMENN Controller Layer based on the intelligent PSO algorithm.

\section{A. Cognition Path Planning Layer:}

The aim of this layer is to collect the information of the environment then plan the desired path to the mobile robot from the starting point to the target point in the environment with a collision-free navigation. To achieve the operation of this layer, a model of the mobile robot and the dimensions of the obstacle are needed in order to avoid the accident between the platform of the mobile robot and the static obstacle. Then the proposed square road map algorithm is used to plan the short and smooth distance of the desired path from the starting point to the target point with obstacles avoidance and generate the optimal desired path equation. The proposed Square Road Map (SRM) methodology is classified as a classical method in the case of static obstacles and it includes road map, mathematical programming, and cell decomposition. 
Therefore, the mobile robot model and the dimension of the obstacle as square shape are needed in order to avoid the collision between the static obstacle and the platform as the distance between two square obstacles becomes greater than the width of the mobile robot platform, as shown in Fig. 3. The optimal desired path equation is generated based on the proposed SRM algorithm that can be described in a flowchart, as shown in Fig. 4.

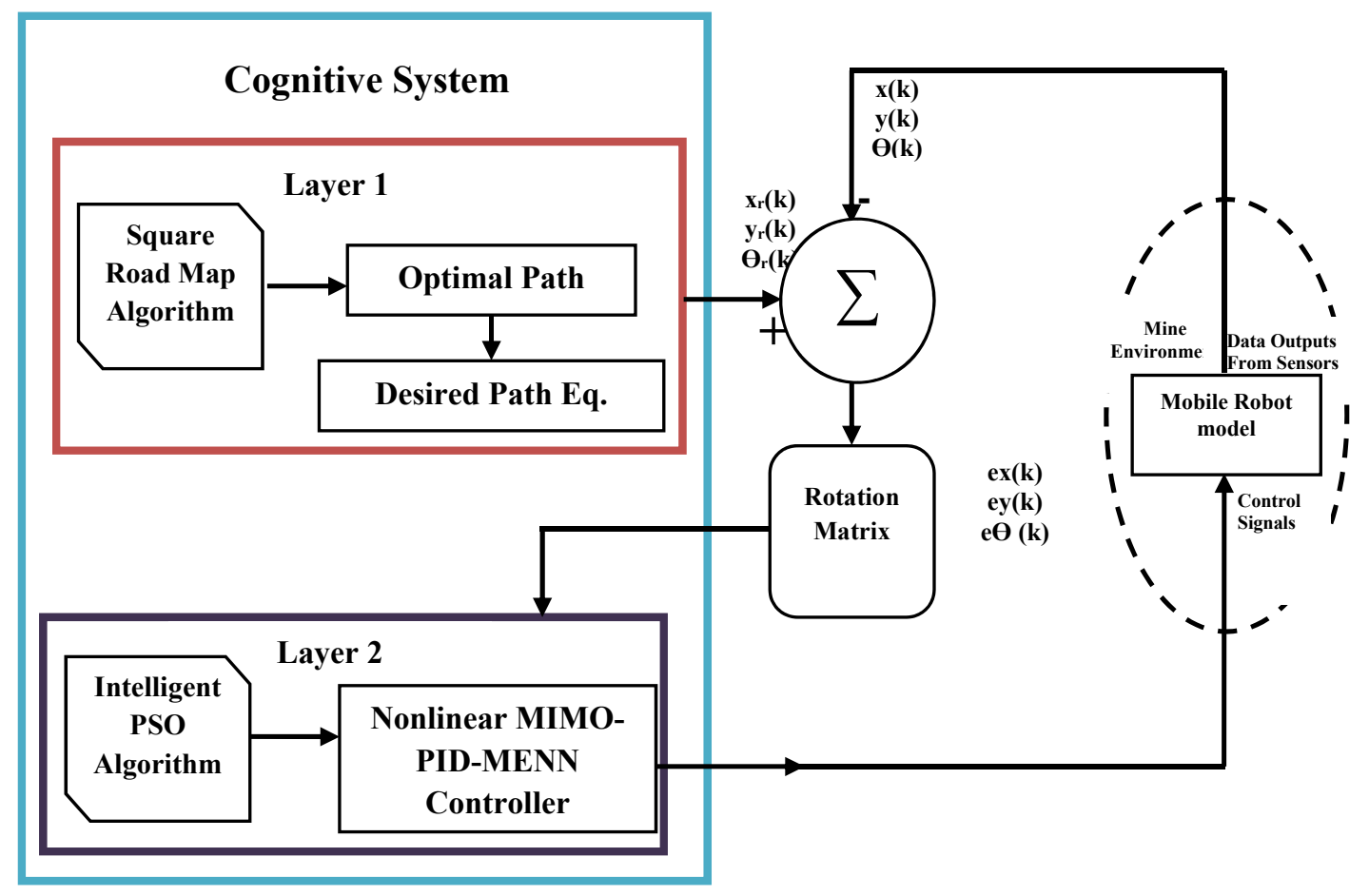

FIG 2: THE COGNITIVE SYSTEM STRUCTURE FOR THE MOBILE ROBOT SYSTEM.

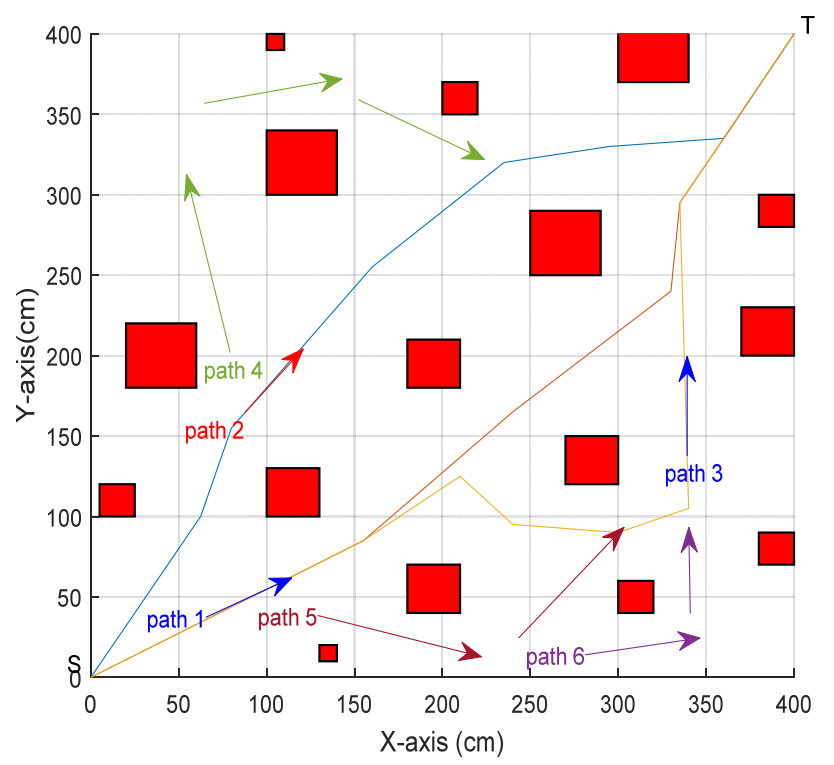

FIG 3. THE GENERATED DESIRED PATH FOR MOBILE ROBOT BASED ON THE PROPOSED SRM ALGORITHM. 


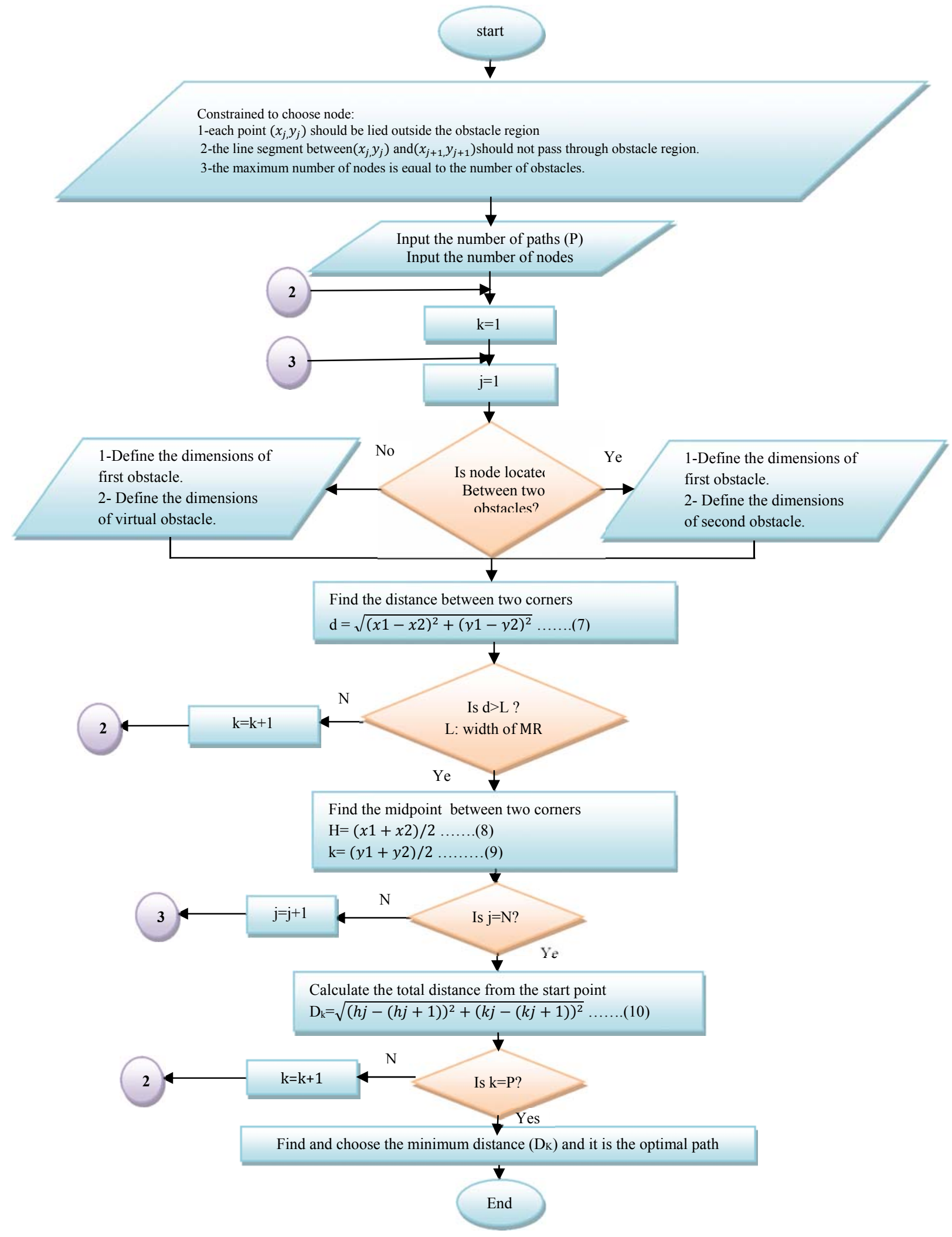

FIG. 4. SRM METHOD FLOWCHART. 
In this work, the environment area of the mobile robot is $400 \times 400 \mathrm{~cm} 2$ and the mobile robot can move in a limited 2 dimensions $(\mathrm{x}, \mathrm{y})$ throughout the starting point to the target point with square shape static obstacles that are distributed randomly in the mobile robot's environment.

\section{B. Nonlinear MIMO-PID-MENN Controller Layer:}

In this section, the proposed nonlinear MIMO-PID-MENN controller structure for the wheeled mobile robot, which is based on the modified Elman recurrent neural network and the PID control equation, is illustrated as shown in Fig. 5. The feedback torque control action is very important in the cognitive system because it is necessary to track the desired path as well as to minimize the tracking pose error of the mobile robot when the actual pose of the mobile robot is drifted from the desired path in the environment. The structure of the nonlinear MIMO-PID-MENN controller consists of four layers. Each of these layers has its own operation as demonstrated below [12], [13]:

- Input Layer: it works as a buffer that transfers the data without any modification.

- Hidden layer: it is the active layer with the non-linear activation functions.

- Context layer: it is a memory layer without activation functions.

- Output layer: it represents a linear collector unit which adds all the fed signals.

The modified Elman neural network weights notation are: $\mathrm{kp}_{\mathrm{x}, \mathrm{y}, \theta}, \mathrm{ki}_{\mathrm{x}, \mathrm{y}, \theta}$ and $\mathrm{kd}_{\mathrm{x}, \mathrm{y}, \theta}$ hidden layers weights. $V c_{1,2}$ : Context layers weight. $L_{i}$ : Linear node as a scaling factor and it is equal to $1 . H$ : Sigmoid nonlinear node. $h_{c}^{o}(k)$ : Context unit output. $h(k)$ : Hidden unit. $\alpha$ : Self-connections feedback gain which is represented randomly between ( 0 and 1$). \beta$ : Connection weight from the hidden layer to the context layer which is represented randomly between $(0$ and 1$)$.

The capabilities of the modified Elman neural network structure give the structure of the controller many abilities such as: fast learning; high adaptation performance; high order control performance and this is due to the context units in MENN which memorize the previous activations of the hidden units; good dynamic characteristic; no output oscillation and strong robustness performance due to the self-connections in the context units which increase the order of the controller model.

The control law of the right wheel torque and left wheel torque for the mobile robot platform depends on the $\mathrm{x}-\mathrm{y}$ position errors and the orientation error as in Eqs. (11) and (12), respectively.

$$
\begin{gathered}
\tau_{R}(k)=o_{x}-o_{y}+h_{c 1}(k) V_{C 1} \\
\tau_{L}(k)=o_{\theta}-o_{y}+h_{c 2}(k) V_{C 2}
\end{gathered}
$$

Where:

$$
h_{c}^{o}(k)=\alpha h_{c}^{o}(k-1)+\beta \tau_{R . L}(K-1)
$$

The modified Elman neural network outputs are based on the nonlinear relationship of the Sigmoid activation function given in Eq. (14).

$$
O_{x, y, \theta}=\frac{2}{1-\exp ^{- \text {net }_{x, y, \theta}}}-1
$$

The summation of each node can be written as follows:

$$
\begin{aligned}
& n e t_{x}(k)=k p e_{x}(k)+k i\left(e_{x}(k)+e_{x}(k+1)\right)+k d\left(\left(e_{x}(k)-e_{x}(k+1)\right)\right. \\
& n e t_{y}(k)=k p e_{y}(k)+k i\left(e_{y}(k)+e_{y}(k+1)\right)+k d\left(\left(e_{y}(k)-e_{y}(k+1)\right)\right. \\
& n e t_{y}(k)=k p e_{\theta}(k)+k i\left(e_{\theta}(k)+e_{\theta}(k+1)\right)+k d\left(\left(e_{\theta}(k)-e_{\theta}(k+1)\right)\right.
\end{aligned}
$$

Where $e_{x}(k), e_{y}(k)$ and $e_{\theta}(k)$ are the error inputs signals. 
Therefore, we needed eleven weight parameters of the controller and we used the PSO algorithm to tune these parameters as in Eqs.(18) and (19):

$$
\begin{aligned}
& V_{i}^{-k+1}=\Omega \Delta V_{i}^{-K}+c_{1} r_{1}\left(\text { pbest }_{i}^{k}-V_{i}^{-K}\right)+c_{2} r_{2}\left(\text { gbest }^{k}-V_{i}^{-K}\right) \\
& V_{i}^{-k+1}=V_{i}^{-k}+\Delta V_{i}^{-k+1}
\end{aligned}
$$

where:

$\Delta V_{i}^{-K+1}$ is the $i^{\text {th }}$ particle's velocity $(k p, k i, k d, V c)$ at $k^{\text {th }}$ iteration; $V_{i}^{k}$ is the $i^{\text {th }}$ particle's position ( $k p, k i, k d, V c$ ) at $k^{\text {th }}$ iteration; $\Omega$ : is the inertia weight factor which equals to 0.73 in order to decrease the iterations number of the evaluation function; $c_{1}$ and $c_{2}$ are the positive values that equal to 1.49 because $\left(c_{1}+c_{2}\right)<4\left[1,5,6\right.$ and 9]; $r_{1}$ and $r_{2}$ are random values ( 0 to 1$) ;$ pbest $_{i}$ is the $i^{\text {th }}$ particle's best previous weight; gbest $_{d}$ is the best particle from the overall population.

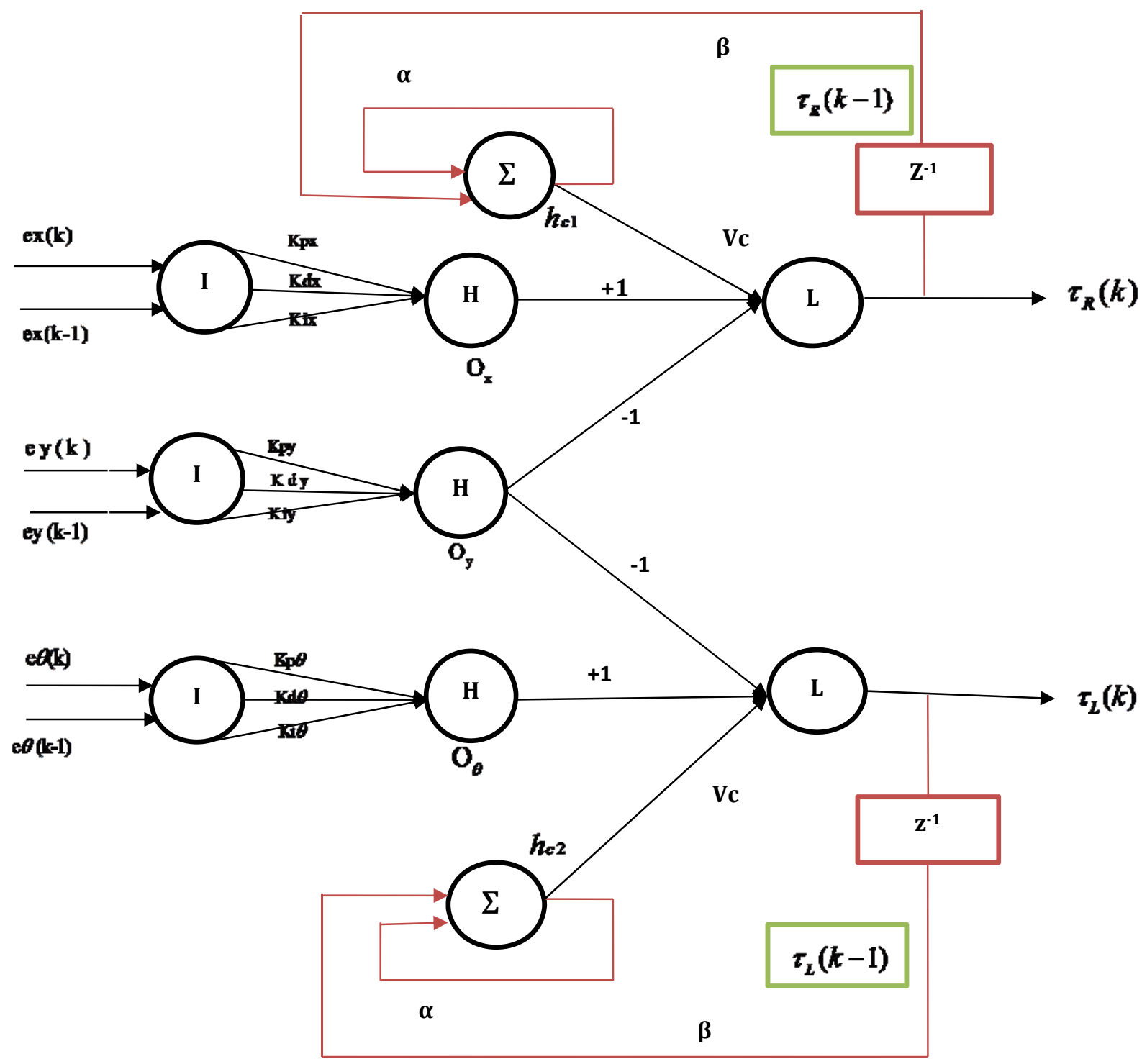

FIG. 5. THE PROPOSED NONLINEAR MIMO-PID-MENN CONTROLLER STRUCTURE. 
In this work, the mean square error function is used as in Eq. (20) to evaluate the performance of the proposed controller.

$$
M S E=\frac{1}{\mathrm{~N}_{\mathrm{it}}} \sum_{i=1}^{\mathrm{N}_{\mathrm{it}}}\left[\left(x_{\text {ref }}(i)-x(i)\right)^{2}+\left(y_{\text {ref }}(i)-y(i)\right)^{2}+\left(\theta_{\text {ref }}(i)-\theta(i)\right)^{2}\right.
$$

$\mathrm{N}_{\mathrm{it}}$ : is the number of iterations.

\section{NUMERICAL SIMULATION RESULTS}

The MATLAB package is used as a numerical simulation " $m$.file" to verify the cognition system based on the proposed square road map algorithm and the proposed nonlinear MIMO-PID-MENN controller for the mobile robot in order to plan and track the desired path with free-navigation. The specifications of the Eddie mobile robot platform are taken from [14 and 15] in terms of Mass=12 kg; inertia of the mobile robot $\mathrm{I}=1.536 \mathrm{~kg} \cdot \mathrm{m}^{2}$; the radius of the wheel $\mathrm{r}=0.075 \mathrm{~m}$ and the distance between the left and right wheels $\mathrm{L}=0.39 \mathrm{~m}$ that are used in Eq. (6) with a sampling time equals to $0.2 \mathrm{sec}$. The first step in the cognition system is carried out by the first layer which is the proposed SRM methodology in order to get the optimal desired path. The second step is achieved by the second layer which is the path tracking based on the nonlinear MIMO-PID-MENN controller with the PSO algorithm. The PSO algorithm is used in this work to get and tune the control parameters of the proposed nonlinear controller which leads to generate the best torque action that leads to minimize the tracking pose error of the mobile robot platform. The PSO parameters can be defined as in Table. I for each case. Each particle has 11 weights because there are eleven parameters of (MIMO-PID-MENN) controller and $r_{1}$ and $r_{2}$ are random values between 0 and 1 .

TABle. I: The PSO PARAMETERs For COGNition Structure.

\begin{tabular}{cccc}
\hline Parameters & Case Study I & Case Study II & Case Study III \\
\hline Number of The population of particle & 25 & 20 & 25 \\
Number of the iterations & 20 & 20 & 25 \\
Acceleration constants c1 and c2 & 1.49 & 1.49 & 1.49 \\
Weight factor & 0.73 & 0.73 & 0.73
\end{tabular}

Case Study I: The mobile robot has an initial pose of $q(0)=[0.0,20.0] \mathrm{cm}$. The proposed SRM method was applied in order to get the optimal path shown in Fig. 6.

The cognitive system has generated three paths to avoid the static obstacles as a first step, then the distances of these three paths between the starting point and the target point are $(589.373,605.292$ and 694.516$) \mathrm{cm}$, respectively based on Eq. (10), so path1 is the optimal path because it has the shortest distance, specifically $(589.373) \mathrm{cm}$. The second step is achieved by obtaining the desired path equation for the optimal path by using the fitting function which was obtained to form the desired path equation as follows:

$$
y(x)=1.442 \times 10^{-8} x^{4}-7.803 \times 10^{-6} x^{3}+0.002621 x^{2}+0.2868 x-0.1028
$$

Therefore, the third step in the cognition system is made by applying the nonlinear MIMO-PID-MENN controller based on the PSO algorithm to make the mobile robot track the desired path based on the desired path equation, as shown in Fig. 7. The excellent tracking performance and the free-navigation for the mobile robot compared with other nonlinear PID controller results as in [6], [14-17] are confirmed in terms of number of iterations and the MSE with different types of tuning control algorithms. Fig. 8 shows the online cost function behaviour for the proposed cognitive system that minimizes the pose error for the motion of the mobile robot during 400 samples which leads to improve the performance of the proposed controller. Fig. 9 shows the smooth left and right torque control actions generated from the proposed MIMO-PID- 
MENN controller without the saturation state (3.07 N.m) which makes the mobile robot follow the desired path equation.

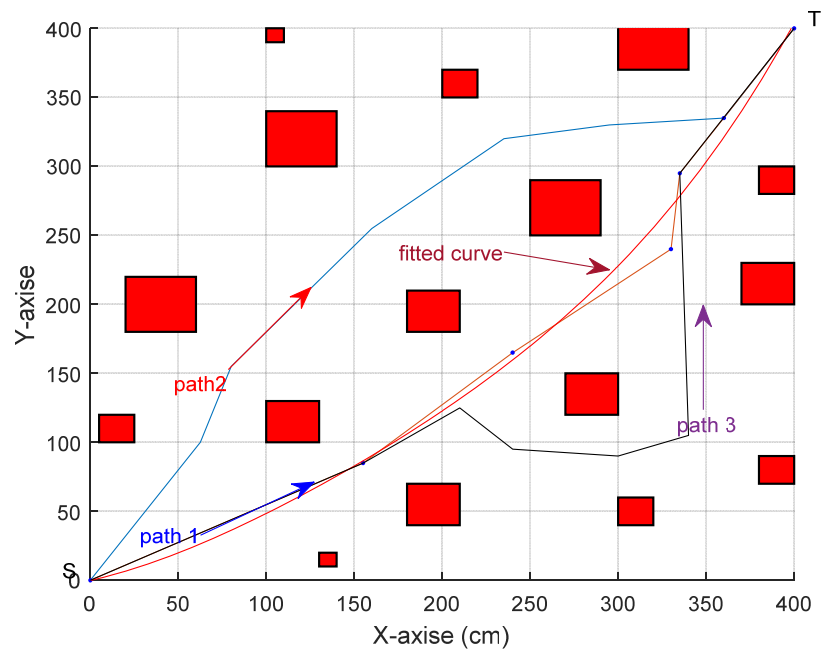

FIG. 6. THE OPTIMAL PATH GENERATED FOR CASE I.

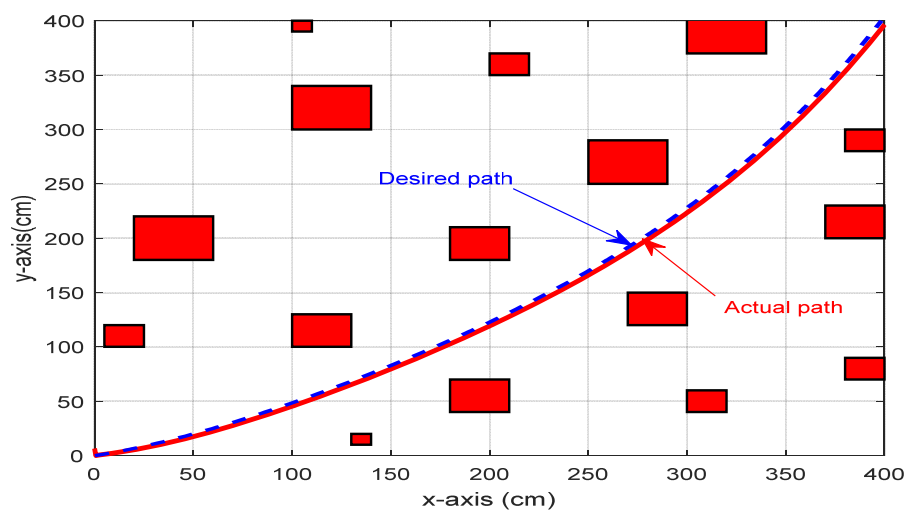

FIG. 7. THE REFERENCE PATH EQUATION AND THE ACTUAL PATH FOR THE MOBILE ROBOT.

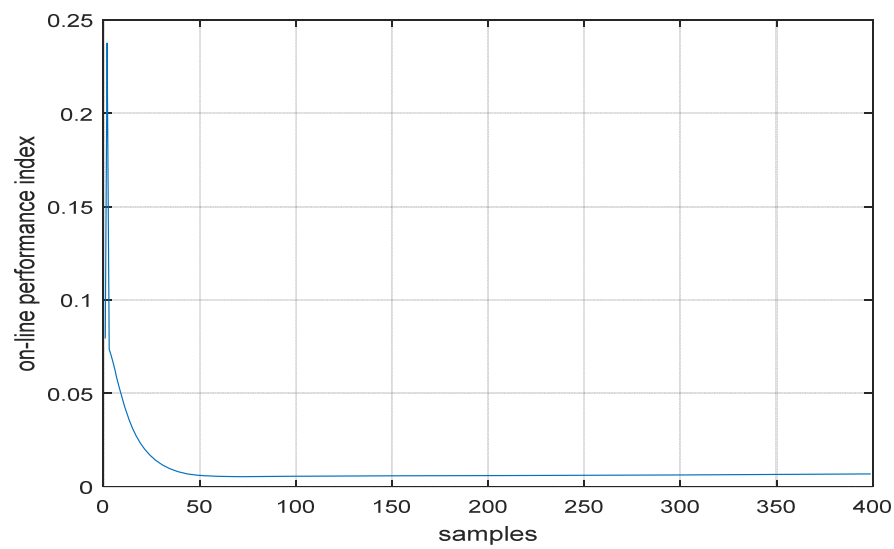

FIG. 8. ON-LINE PERFORMANCE INDEX.

Received 6 Feb 2018; Accepted 9 May 2018 


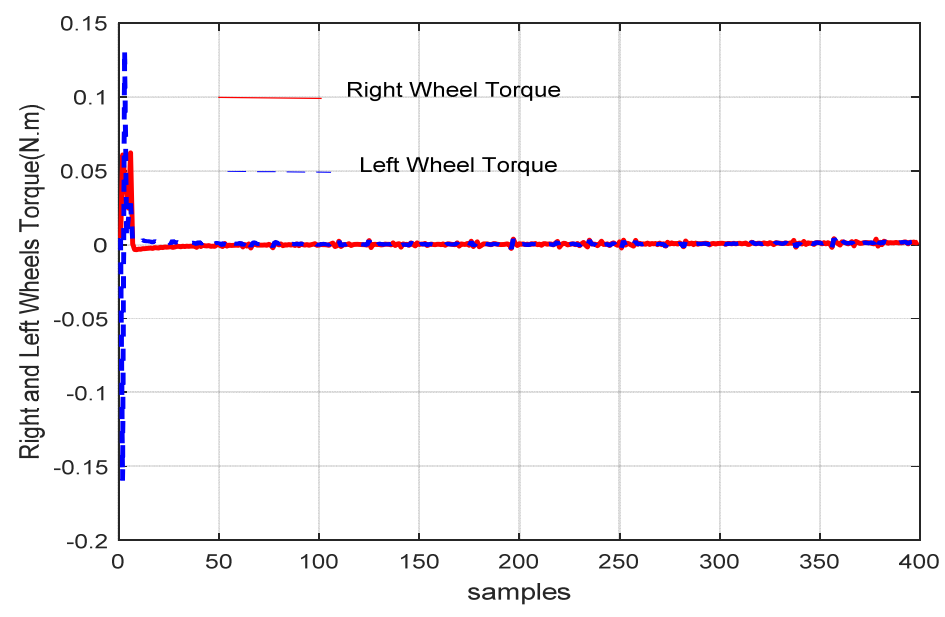

FIG. 9. TORQUE CONTROL ACTION.

The linear and angular velocities responses for the non-holonomic wheel mobile robot are smooth and they did not have a sharp-spikes action, as shown in Fig. 10.

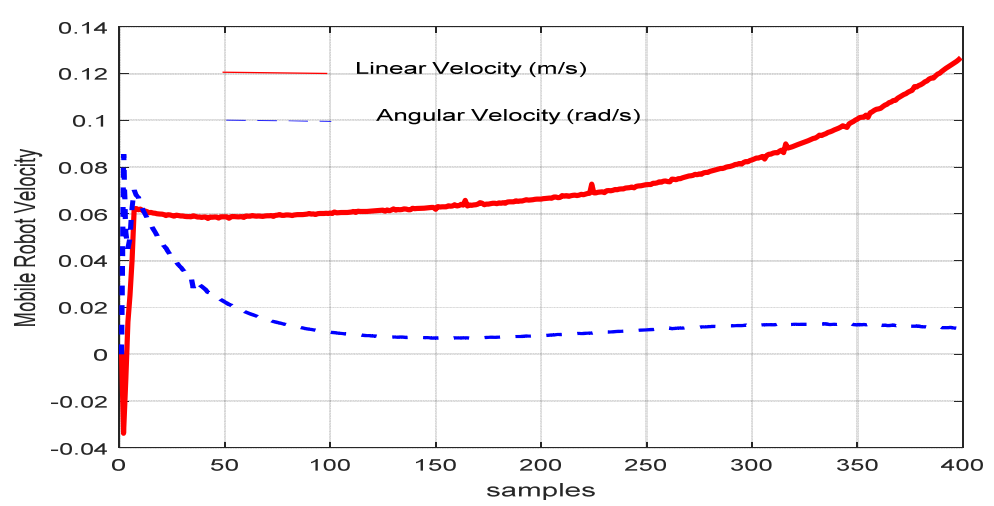

FIG. 10. THE LINEAR VELOCITY AND THE ANGULAR VELOCITY OF THE MOBILE ROBOT.

To confirm that the proposed controller has a high robustness and strong adaptation performances, bounded dynamic disturbances, which are taken from [14-16], are added to the system: $\overline{\tau d}=\left[\begin{array}{ll}0.01 \sin (2 t) & 0.01 \sin (2 t)\end{array}\right]^{T}$. The system has reduced the effect of these disturbances by showing the minimum tracking pose error for the mobile robot platform.

Case Study II: The mobile robot has an initial pose of $q(0)=[0.0,380.0] \mathrm{cm}$. The proposed SRM method was applied in order to get the optimal path, as shown in Fig. 12 based on the flowchart of the SRM algorithm which generated three paths to avoid the static obstacles, then the distances of these three paths between starting point to target point are $(592.439,620.016$ and 627.287$) \mathrm{cm}$, respectively based on Eq. (10), so path1 is the optimal path because it has the shortest distance of $(592.439) \mathrm{cm}$. Then the desired path equation was obtained as follows:

$$
y(x)=5.428 \times 10^{-8} x^{4}-6.585 \times 10^{-5} x^{3}+0.02307 x^{2}-3.174 x+399.8
$$


$\mathbf{a}$

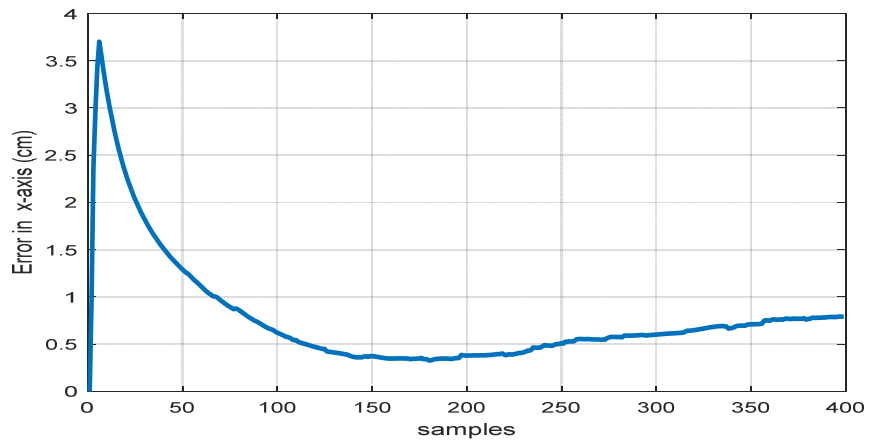

b

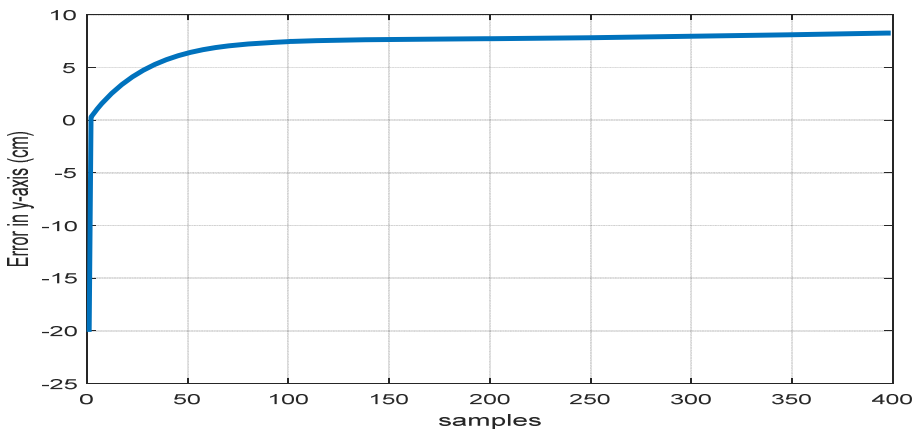

c

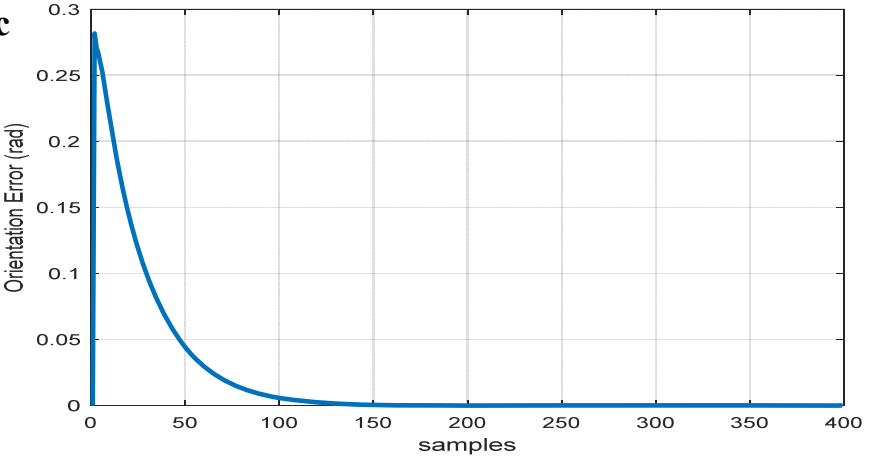

FIG. 11. POSE ERROR OF THE MOBILE ROBOT: A) ERROR IN X-AXIS; B) ERROR IN Y-AXIS; C) ORIENTATION ERROR.

Fig. 13 shows that the mobile robot follows the desired path equation. The ability of the proposed controller response was shown by generating smooth left and right torques actions without a saturation state to follow the reference path equation in minimum time, as shown in Fig. 14. The linear and angular velocities responses for the non-holonomic wheel mobile robot are smooth and they did not have high spikes, as shown in Fig. 15. 


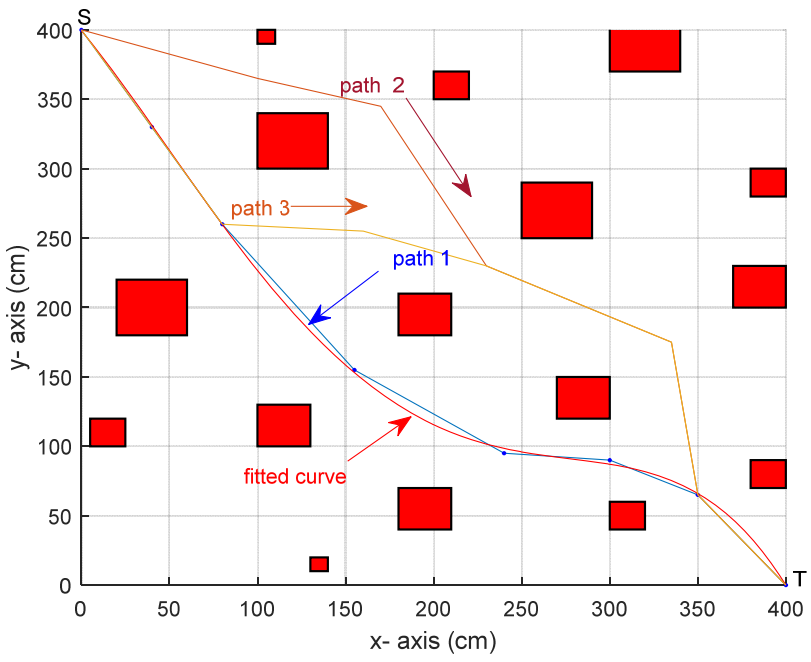

Fig. 12. THE OPTIMAL PATH GENERATED FOR CASE II.

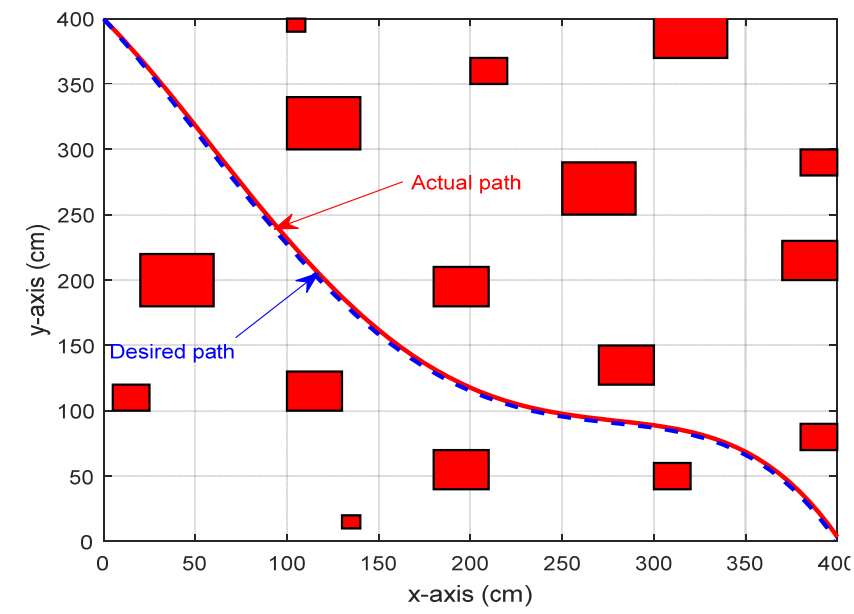

FIG. 13. THE REFERENCE PATH EQUATION AND THE ACTUAL PATH FOR THE MOBILE ROBOT.

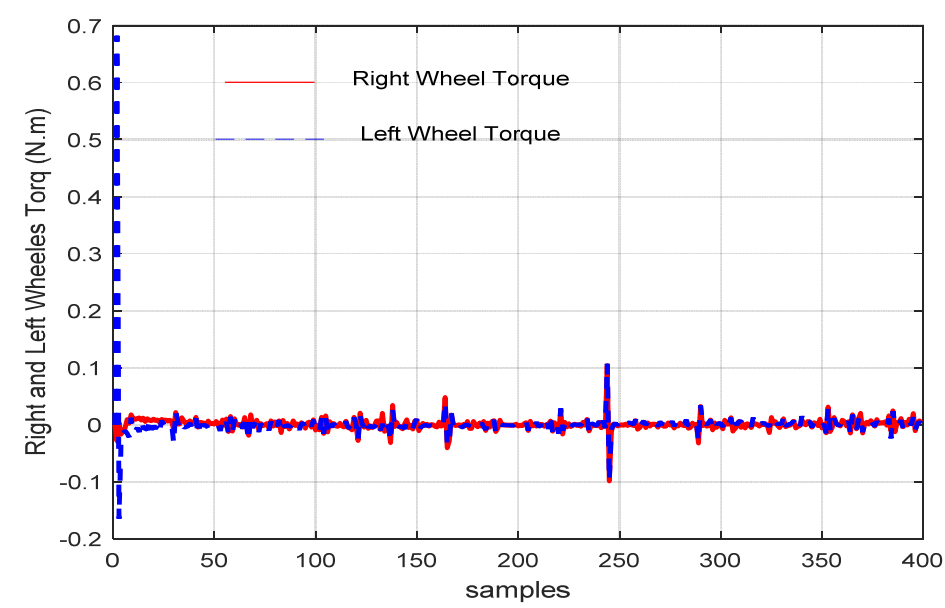

FIG. 14. TORQUE CONTROL ACTION. 
Case Study III: To verify the effectiveness of the proposed SRM method, Fig. 16 shows that the SRM method has added some virtual obstacles in order to achieve the steps for planning the path for the mobile robot which has an initial pose of $q(0)=[0.0,20.0] \mathrm{cm}$. Then, three paths were generated to avoid the static obstacles and the distances of these three paths between starting point to target point are $(591.639,613.969$ and 693.7423$) \mathrm{cm}$, respectively based on Eq. (10), so path1 is the optimal path because it has the shortest distance of (591.639) $\mathrm{cm}$. Then the desired path equation for the optimal path is given in equation (23) by using the following fitting function.

$$
y(x)=3.636 \times 10^{-8} x^{4}-2.415 \times 10^{-5} x^{3}+0.006194 x^{2}+0.0685 x-0.1068
$$

Figure 17 shows that the platform of the mobile robot follows the desired path and Fig. 18 shows the smooth torque control action without a saturation control action state that was generated from the proposed controller. The linear and angular velocities responses for the non-holonomic wheeled mobile robot are smooth and without sharp spikes, as shown in Fig. 19.

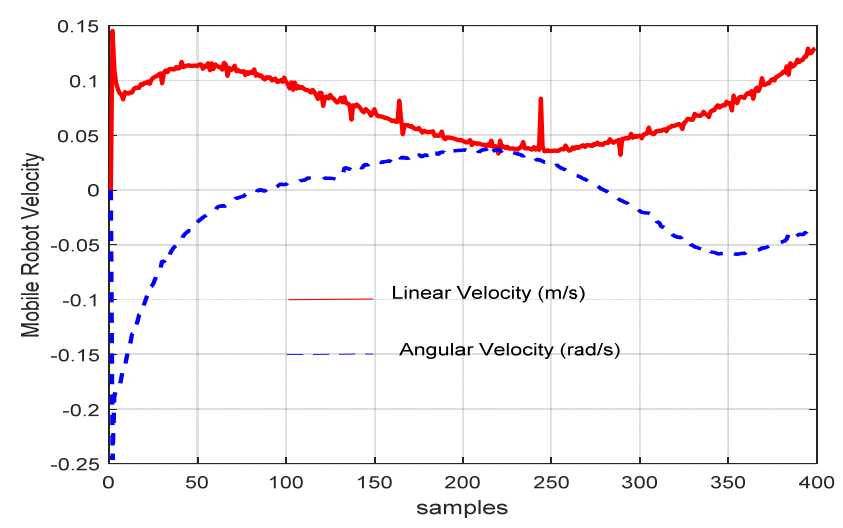

FIG. 15. THE LINEAR VELOCITY AND THE ANGULAR VELOCITY OF THE MOBILE ROBOT.

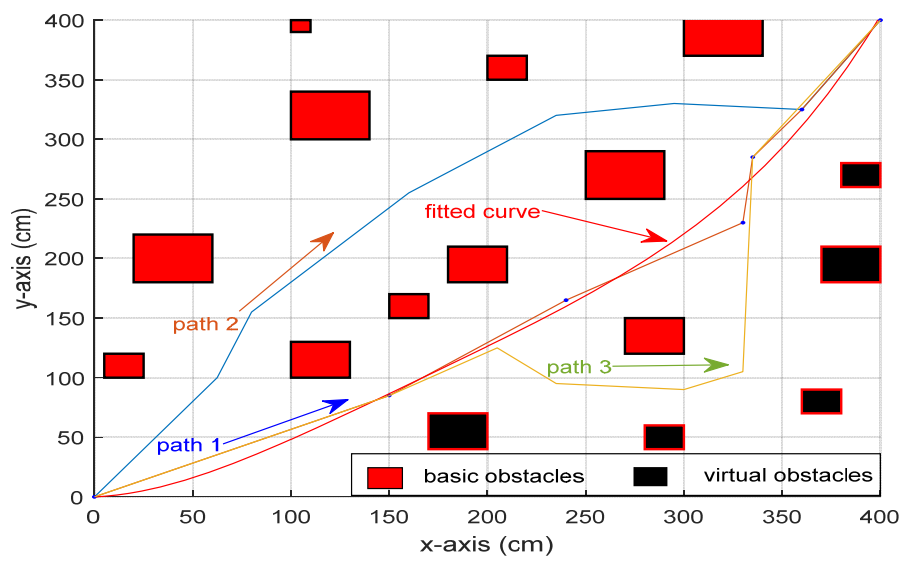

FIG. 16. THE OPTIMAL PATH GENERATED FOR CASE III. 


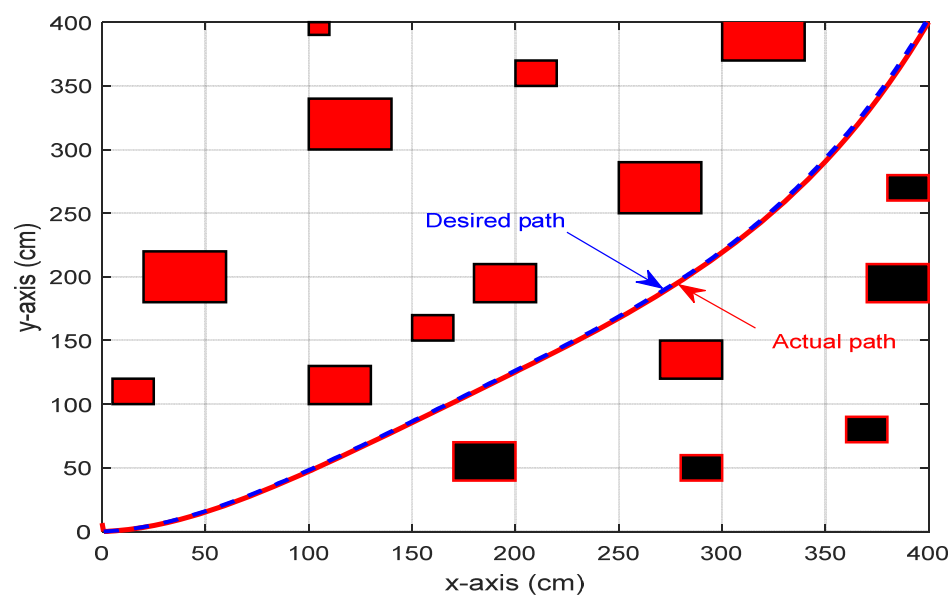

FIG. 17. THE REFERENCE PATH EQUATION AND THE ACTUAL PATH FOR THE MOBILE ROBOT.

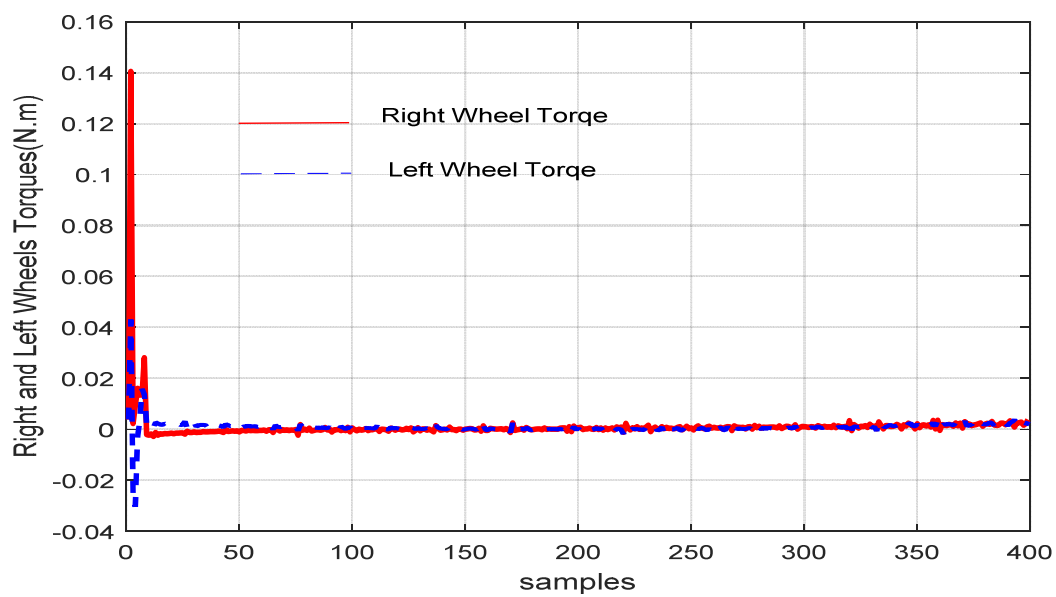

FIG. 18.TORQue CONTROL ACTION.

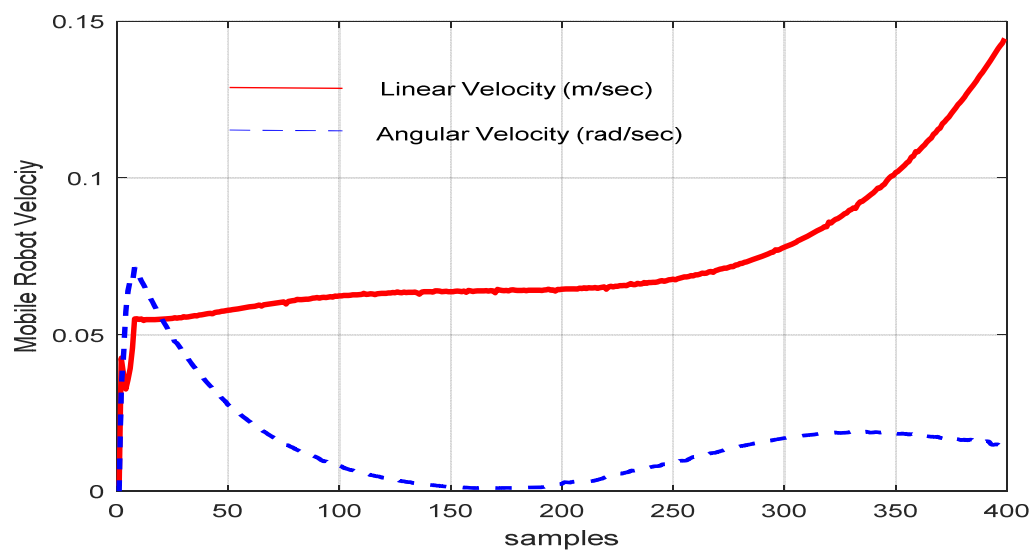

Fig. 19. THE LINEAR VELOCITY AND THE ANGULAR VELOCITY OF THE MOBILE ROBOT. 


\section{CONCLUSIONS}

The numerical simulation results of the proposed cognitive system based on the square road map algorithm and the nonlinear MIMO-PID-MENN controller with PSO algorithm are presented in this paper for the path planning of the mobile robot and trajectory tracking which show the following capabilities:

- Highly accurate short distance planning is obtained by using the square road map algorithm with a collision free path from the starting position to the target position for the mobile robot during working in obstacles environment.

- Fast on-line tuning control parameters of the proposed controller by using the PSO algorithm in order to generate the best torque control action without a saturation state.

- An excellent tracking of the desired reference path with a minimum pose error is obtained.

- Strong adaptability performance when changing the initial pose for the mobile robot in each case.

- High robustness performance of the proposed controller when adding the bounded disturbances to the mobile robot.

\section{REFERENCES}

[1] M. S. Alam, M. U. Rafique, and M. U. Khan "Autonomous Robot Path Planning Using Particle Swarm Optimization in Static and Obstacle Environment". International Journal of Computer Science and Electronics Engineering (IJCSEE) Volume 3, No. 3, pp.253-257, (2015).

[2] M. Asif, M. J. Khan, M. Rehan and M. Safwan,'Feedforward and Feedback Kinematics Controller for Wheeled Mobile Robot Trajectory Tracking”. Journal of Automation and Control Engineering, Vol. 3, No.3, pp.178-182, (2015).

[3] R. k. Panda and B.B. Choudhury “An Effective Path Planning of Mobile Robot Using Genetic Algorithm”, Proceeding of the IEEE International Conference on Computational Intelligence \& Communication Technology, pp. 287-291, (2015).

[4] K. Ming , "Solving Path Planning Problem Based on Ant Colony Algorithm",Proceeding of The $29^{\text {th }}$ IEEE International Conference on Chinese Control and Decision Conference, pp. 5391-5395, (2017) .

[5] M. k. Rath and B. B. Deepak, "PSO based system architecture for Path Planning of Mobile Robot in Dynamic Environment". Proceedings of the IEEE Global Conference on Communication Technologies, pp. 797-801, (2015).

[6] K. E. Dagher and A. S. Al-Araji, "Design of a Nonlinear PID Neural Trajectory Tracking Controller for Mobile Robot based on Optimization Algorithm”. Engineering \& Technology Journal. Vol. 32, No. 4, pp. 973-986.(2014).

[7] M. H. Al-Dahhan and M. M. Ali, "Path tracking control of a mobile robot using fuzzy logic". Proceedings of the $13^{\text {th }}$ IEEE International Conference on Multi-Conference on Systems, Signals and Devices, pp. 82-88, (2016).

[8] M. K. Hilfi and D. Cheng, "Mobile Robot- Dynamic Model Controlling using Wavelet Network". International Journal of Computer Applications. Vol. 95, No. 8, pp. 41-45, (2014).

[9] A. S. Al-Araji and N. Yousif, "Design of a Nonlinear Controller for Wheeled Mobile Robot based on Cognitive On-Line Hybrid Bees-PSO Optimization Algorithm". Proceedings of the 1st IEEE International Conference on Recent Trends of Engineering Sciences and Sustainability, Baghdad University, Iraq, pp. 307-312, (2017).

[10] S. M. Swadi, M. A. Tawfik, E. N. Abdulwahab and H. Almgotir-Kadhim,"Fuzzy-Backstepping Controller Based on Optimization Method for Trajectory Tracking for Wheeled Mobile Robot". The $18^{\text {th }}$ International Conference on Computer Modeling and Simulation, pp. 91-97, (2016).

[11] Y. Koubaa, M. Boukattaya and T. Dammak," Adaptive Sliding-Mode Dynamic Control for Path Tracking of Nonholonomic Wheeled Mobile Robot". Journal of Automation and Systems Engineering, Vol. 9, No. 2, pp. 119-131, (2015).

[12] A. S. Al-Araji, M. Abbod, and H. Al-Raweshidy, "Design of a neural predictive controller for nonholonomic mobile robot based on posture identifier". Proceedings of the IASTED International Conference Intelligent Systems and Control (ISC 2011), Cambridge, United Kingdom, pp. 198-207, (2011).

[13] A. S. Al-Araji, M. Abbod, and H. Al-Raweshidy, "Neural autopilot predictive controller for nonholonomic wheeled mobile robot based on a pre-assigned posture identifier in the presence of disturbances". The $2^{\text {nd }}$ International Conference on Control, Instrumentation and Automation (ICCIA), pp. 326-331, (2011).

[14] A. S. Al-Araji and L. T. Rasheed, "Design of a Nonlinear Fractional Order PID Neural Controller for Mobile Robot Based on Particle Swarm Optimization”. Engineering \& Technology Journal. Vol. 34, No. 12, pp. 2318-2333, (2016). 
[15] A. S. Al-Araji and L. T. Rasheed," A Cognitive Nonlinear Fractional Order PID Neural Controller Design for Wheeled Mobile Robot based on Bacterial Foraging Optimization Algorithm”. Engineering \& Technology Journal. Vol. 35, No. 2, pp. 289300, (2017).

[16] A. S. Al-Araji, "A Comparative Study of Various Intelligent Algorithms Based Nonlinear PID Neural Trajectory Tracking Controller for the Differential Wheeled Mobile Robot Model”. Journal of Engineering. Vol. 20, No. 5, pp. 44-60, (2014).

[17] A. S. Al-Araji, "A Cognitive PID Neural Controller Design for Mobile Robot Based on Slice Genetic Algorithm”. Engineering \& Technology Journal. Vol. 33, No. 1, pp. 208-222, (2015). 